\title{
Is surface-based orientation influenced by a proportional relationship of shape parameters?
}

\author{
Bradley R. Sturz $・$ Kent D. Bodily
}

Published online: 21 May 2011

(C) Psychonomic Society, Inc. 2011

\begin{abstract}
We investigated the extent to which parameters of environmental shape - namely the major and minor principal axes of space which pass through the centroid and approximate length and width of the entire space, respectively, were subject to similar psychophysical principles as those involved in distance discriminations. We developed an orientation task that allowed us to manipulate the ratio of the major to the minor principal axes of an enclosure during training and control for orientation by alternative cues other than principal axes such as wall lengths or corner angles during testing. Participants trained in an environment with a larger hypothetical discriminability ratio allocated more responses to locations specified by the principal axes of space across novel enclosure types compared to a group trained with a smaller hypothetical discriminability ratio. Results suggest that psychophysical principles may operate on the discrimination of environmental shape parameters and delineate a potential mechanism for experiential and developmental changes in orientation ability.
\end{abstract}

Keywords Psychophysical principle · Principal axis . Orientation $\cdot$ Hexagon $\cdot$ Geometry

For centuries, psychophysical principles have guided investigations into the mechanisms that underlie stimulus

B. R. Sturz $(\bowtie)$

Department of Psychology, Armstrong Atlantic State University,

229 Science Center, 11935 Abercorn Street,

Savannah, GA 31419, USA

e-mail: bradley.sturz@armstrong.edu

K. D. Bodily

Department of Psychology, Georgia Southern University,

Statesboro, GA, USA discrimination (for a review, see Gescheider, 1997). As perhaps the most well-known, Weber's law specifies an amount of stimulus change that must occur in order for the now-modified stimulus to be considered psychologically distinct (for a review, see Fechner, 1860/1966). Proportional to the amount of the original stimulus, this just noticeable difference provides an objective physical threshold for the subjective psychological detection of a change in stimulus intensity (e.g., loudness, size, etc.).

For decades, it has been suggested that spatial representations are based on metric relations (Gallistel, 1990). Metric relations that have received considerable attention are based on parameters of environmental shape-namely, the major and minor principal axes of space that pass through the centroid and the approximate length and width of the entire space, respectively (for a detailed mathematical and mechanical definition, see Cheng, 2005; see also Fig. 1). The psychological extraction of the principal axes of space provides the stimuli by which participants are able to assign a sense of direction (i.e., left/right, top/bottom) and allows orientation with respect to the environment (Hermer \& Spelke, 1994; for a review, see Cheng \& Newcombe, 2005; see also Cheng \& Gallistel, 2005; Sovrano \& Vallortigara, 2006; see also Rock, 1973, and Shelton \& McNamara, 2001, for supporting evidence in object memory and layout memory, respectively).

Within a psychophysical framework, we hypothesized that if participants are psychologically extracting the major and minor principal axes of space from an environmental enclosure, the discrimination processes involved may be subject to psychophysical principles similar to those involved in the discrimination of other visual stimuli-specifically, distance discriminations (Cheng, 1988, 1989, 1990; Cheng, Srinivasan, \& Zhang, 1999). We hypothesized that the discriminability (and as a result, the potential usefulness as 


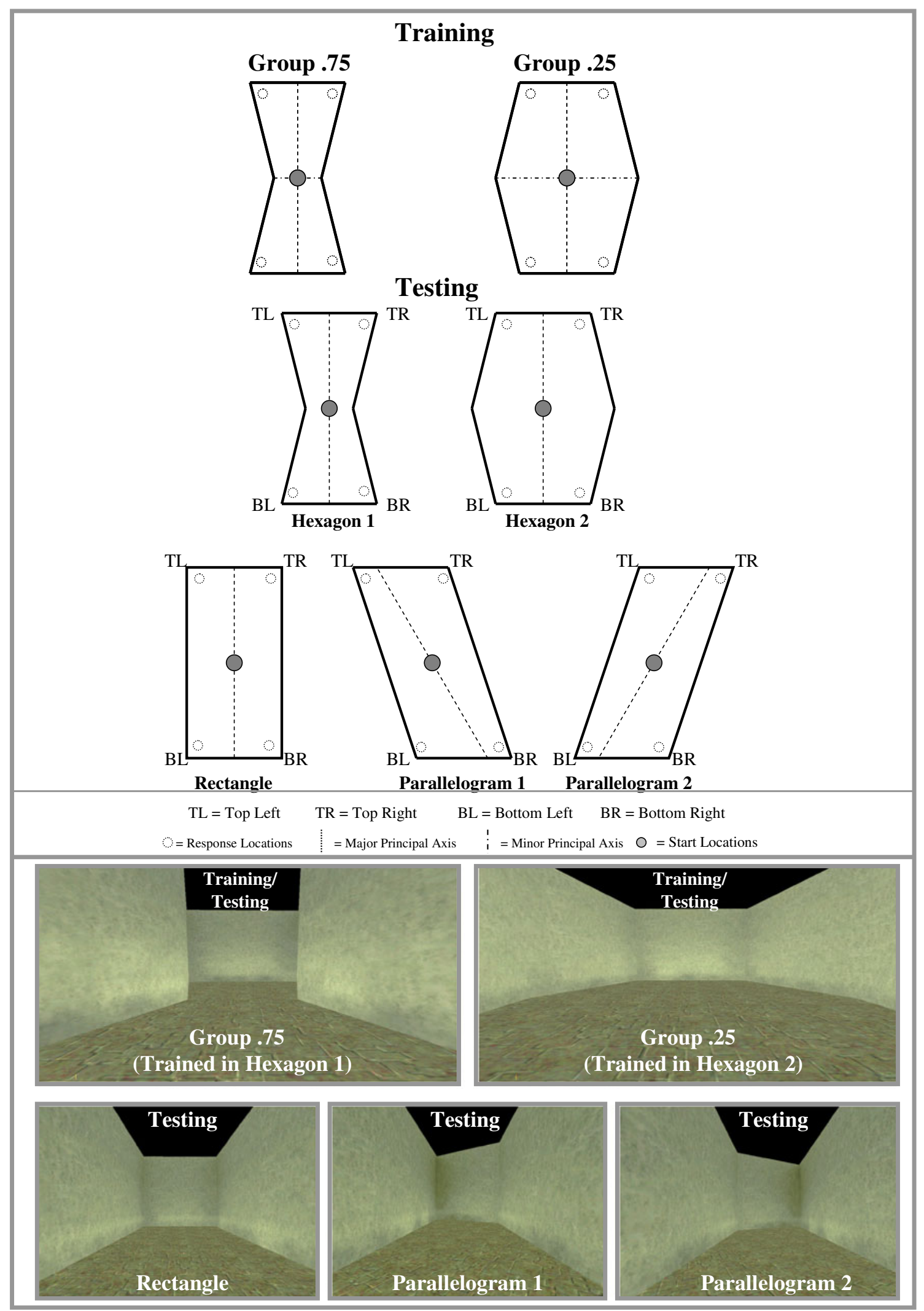

Fig. 1 Top panel: Schematics of training and testing trials for each group. For illustrative purposes, the gray circles mark the position where participants entered the virtual enclosures for all training and testing trials, large unfilled dotted circles indicate response locations, dotted lines represent the major principal axis of space, and dotteddashed lines indicate the minor principal axis of space. Bottom panel: Sample images from the first-person perspective of virtual environment search spaces as seen from the far end of the enclosure 
an orienting cue) of the major principal axis is a function of its relation to the minor principal axis as follows:

length of major principal axis - length of minor principal axis length of major principal axis

As this hypothetical ratio approaches 1, the major and minor principal axes will become more discriminable from each other, but as this hypothetical ratio approaches 0 , the major and minor principal axes will become less discriminable from each other. As a result, the discriminability of the major and minor principal axes (and by extension, their potential usefulness as spatial cues for orientation) would necessarily be enclosure specific.

The purpose of the present experiment was to determine the influence of the discriminability of the major and minor principal axes on the use of the major principal axis as an orienting cue and, by extension, the extent to which psychophysical principles may operate on the discrimination of parameters of environmental shape. Using an established dynamic three-dimensional virtual environment preparation (Sturz, Gurley, \& Bodily, 2011), we trained one group of participants (Group .75) to find locations specified by the principal axis of a hexagon-shaped enclosure in which the aforementioned hypothetical discriminability ratio between the major and minor principal axes was .75 , and we trained another group of participants to find locations specified by the principal axis of a hexagon-shaped enclosure in which the aforementioned hypothetical discriminability ratio between the major and minor principal axes was .25 (see Fig. 1).

Following training, we presented participants in both groups with both hexagons along with a rectangle, a parallelogram, and the parallelogram's mirror equivalent ${ }^{1}$ (see Fig. 1). The unique shape of the training hexagons, coupled with the carefully selected testing enclosures, allowed us to control for orientation by alternative environmental cues such as wall lengths or corner angles (Dawson, Kelly, Spetch, \& Dupuis, 2010; Miller \& Shettleworth, 2007; for a review, see Cheng, 2008) and to assess the extent to which participants utilized the principal axes of the enclosures for orientation. To the extent that psychophysical principles may operate on the discrimination of parameters of environmental shape, this hypothesized

\footnotetext{
${ }^{1}$ The parallelogram's mirror equivalent was presented to provide both groups with the opportunity to respond to locations specified by the principal axis of the parallelograms and/or corner angles in the parallelograms similar to those experienced during training (i.e., acute/ obtuse). This was necessary because training angles differed in the hexagons across groups and participants were randomly assigned to respond to top-left/bottom-right or top-right/bottom-left within group. Performance in these parallelograms was then averaged for results. For details, see the Method and Results sections.
}

discriminability ratio should influence the use of the principal axes of space for orientation. Specifically, participants trained in the enclosure with a larger hypothetical discriminability ratio (i.e., Group .75) should allocate more responses to locations specified by the major (or minor) principal axis of space across enclosures types, as compared with that of participants trained in the enclosure with a smaller hypothetical discriminability ratio (i.e., Group .25).

\section{Method}

\section{Participants}

Thirty-two undergraduate students ( 8 males and 24 females) served as participants. Participants received extra class credit.

\section{Apparatus}

An interactive, dynamic three-dimensional virtual environment was constructed and rendered using Valve Hammer Editor and run on the Half-Life Team Fortress Classic platform. A personal computer, 21-in. flat-screen liquid crystal display (LCD) monitor, gamepad joystick, and speakers served as the interface with the virtual environment. The monitor $(1,152 \times 864$ pixels $)$ provided a firstperson perspective of the virtual environment (see bottom panel, Fig. 1). Participants utilized the joystick on the gamepad to navigate within the environment. Speakers emitted auditory feedback. Experimental events were controlled and recorded using Half-Life Dedicated Server on an identical personal computer.

\section{Stimuli}

Dimensions are length $\times$ width $\times$ height and are measured in virtual units (vu). Five virtual enclosures were created (see Fig. 1): hexagon $1(550 \times 136 \times 260 \mathrm{vu}=\sim 14 \times 3.5 \times$ $6.6 \mathrm{~m})$, hexagon $2(550 \times 412 \times 260 \mathrm{vu}=\sim 14 \times 10.5 \times$ $6.6 \mathrm{~m})$, rectangle $(550 \times 276 \times 260 \mathrm{vu}=\sim 14 \times 7 \times 6.6 \mathrm{~m})$, parallelogram $1(550 \times 276 \times 260 \mathrm{vu}=\sim 14 \times 7 \times 6.6 \mathrm{~m})$, and parallelogram $2(550 \times 276 \times 260 \mathrm{vu}=\sim 14 \times 7 \times 6.6 \mathrm{~m})$. Please note that the width of hexagon 1 was measured at its minimum width (in the middle) and the width of hexagon 2 was measured at its maximum width (also in the middle). Hypothetical discriminability ratios were $.75, .25, .50, .58$, and .58 , respectively. All corner angles were $75^{\circ}$ in hexagon 1 , and all corner angles were $105^{\circ}$ in hexagon 2 . Corner angles in the parallelograms were $60^{\circ}$ for both acute angles and $120^{\circ}$ for both obtuse angles. All angles were $90^{\circ}$ in the rectangle. We delineated four response locations within each enclosure $(48 \times 48 \times 48 \mathrm{vu}=\sim 1.2 \times 1.2 \times 1.2 \mathrm{~m}$; see Fig. 1), but response locations were not visible to participants. 
All surfaces were white in color with the exceptions of the floors (gray tile) and the ceilings (black).

\section{Procedure}

Participants were informed that they should navigate to the location that transported them to the next virtual room and moved via the joystick on the gamepad: $\uparrow$ (forward), $\downarrow$ (backward), $\leftarrow$ (rotated view left), and $\rightarrow$ (rotated view right). Simulated eye height was 68 virtual units $(\sim 1.73 \mathrm{~m})$. Participants selected a location by walking into it. Selection of a rewarded location resulted in auditory feedback (bell sound) and a 7-s inter trial interval (ITI) in which the monitor went black and participants progressed to the next trial. Selection of a non rewarded location resulted in different auditory feedback (buzz sound) and required participants to continue searching.

Training Training consisted of 12 trials. Participants were randomly assigned to one of two groups: Group .75 or Group .25. The group name reflects the ratio of the principal axes within the training hexagons as described above (see also Fig. 1). Gender and number of participants were balanced across groups. Within each group, participants were randomly assigned to either the left or the right side of the major principal axis (i.e., two of the four response locations: top-left/bottom-right or top-right/bottom-left). The locations specified by this side of the major principal axis were then designated as the rewarded locations. Gender and number of participants trained at top-left/bottom-right and top-right/bottom-left were balanced within group. Participants in Group .75 were trained within hexagon 1, and participants in Group .25 were trained within hexagon 2. All participants started each trial in the center of their respective hexagon (marked with a gray circle in Fig. 1). Participants entered their training hexagon at random orientations from $0^{\circ}$ to $270^{\circ}$ in increments of $90^{\circ}$.

Testing Testing consisted of 60 trials composed of 15 fourtrial blocks. Each trial block was composed of 3 training trials and 1 test trial. The location of the test trial was randomized within each block. For each test trial, one of five enclosures was presented: hexagon 1, hexagon 2, rectangle, parallelogram 1, or parallelogram 2. Each enclosure was presented once without replacement until all five had been presented. Each enclosure was presented three times (total of 15 test trials). Participants made one response during test trials, which resulted in no auditory feedback, followed by the 7-s ITI and progression to the next trial. Participants entered all enclosures during testing in the center of the enclosures (marked with gray circles in Fig. 1) at random orientations from $0^{\circ}$ to $270^{\circ}$ in increments of $90^{\circ}$.

\section{Results}

Training

Figure 2 shows the mean proportion of participants' correct first responses plotted by 3-trial blocks for the 12 trials of training. As is shown, participants rapidly learned to respond to their respective trained side of the major principal axis (i.e., correct locations). A two-way mixed analysis of variance (ANOVA) on mean proportion of correct first responses, with group $(.75, .25)$ and block $(1-4)$ as factors, revealed only a main effect of block, $F(3,90)=3.52$, $p<.001$. Neither the effect of group nor the interaction was significant, $F_{\mathrm{S}}<1, p \mathrm{~s}>.3$. Post hoc tests on the block factor revealed that blocks 1 and 2 were significantly different from each other and significantly different from blocks 3 and 4 ( $p$ s $<.05)$; however, blocks 3 and 4 were not significantly different from each other $(p=.24)$. Additionally, block 1 was not significantly different from chance performance [i.e., .5; one-sample $t$-test, $t(31)=-0.38, p>.7$ ], but blocks $2-4$ were each significantly greater than chance performance[one-sample $t$-tests, $t \mathrm{~s}(31)>3.2, p \mathrm{~s}<.01$ ].

\section{Testing}

A two-way mixed ANOVA on mean proportion of responses to locations specified by the trained side of the major principal axis, with group $(.75, .25)$ and enclosure type (hexagon 1 , hexagon 2 , rectangle, parallelograms) as factors, revealed a main effect of group, $F(1,30)=5.24$, $p<.05$, and a main effect of enclosure type, $F(3,90)=$ $7.02, p<.001$. The group $\times$ enclosure type interaction was not significant, $F<1.66, p \mathrm{~s}>.18$. Figure 3 (top panel) shows the main effect of group; overall, participants in Group .75 allocated a greater proportion of responses to the locations specified by the trained side of the major

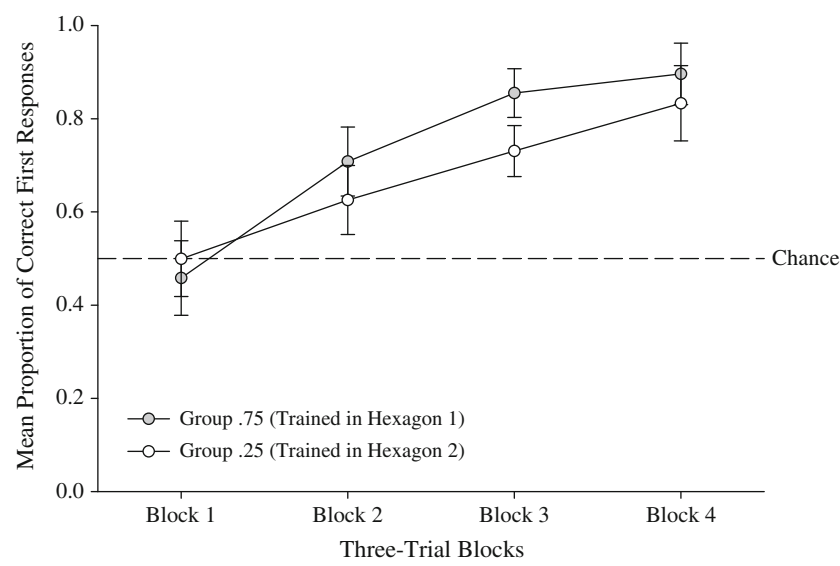

Fig. 2 Mean proportion of participants' correct first responses plotted by 3 -trial blocks for the 12 trials of training. Dashed line represents chance performance. Error bars represent standard errors of the means 

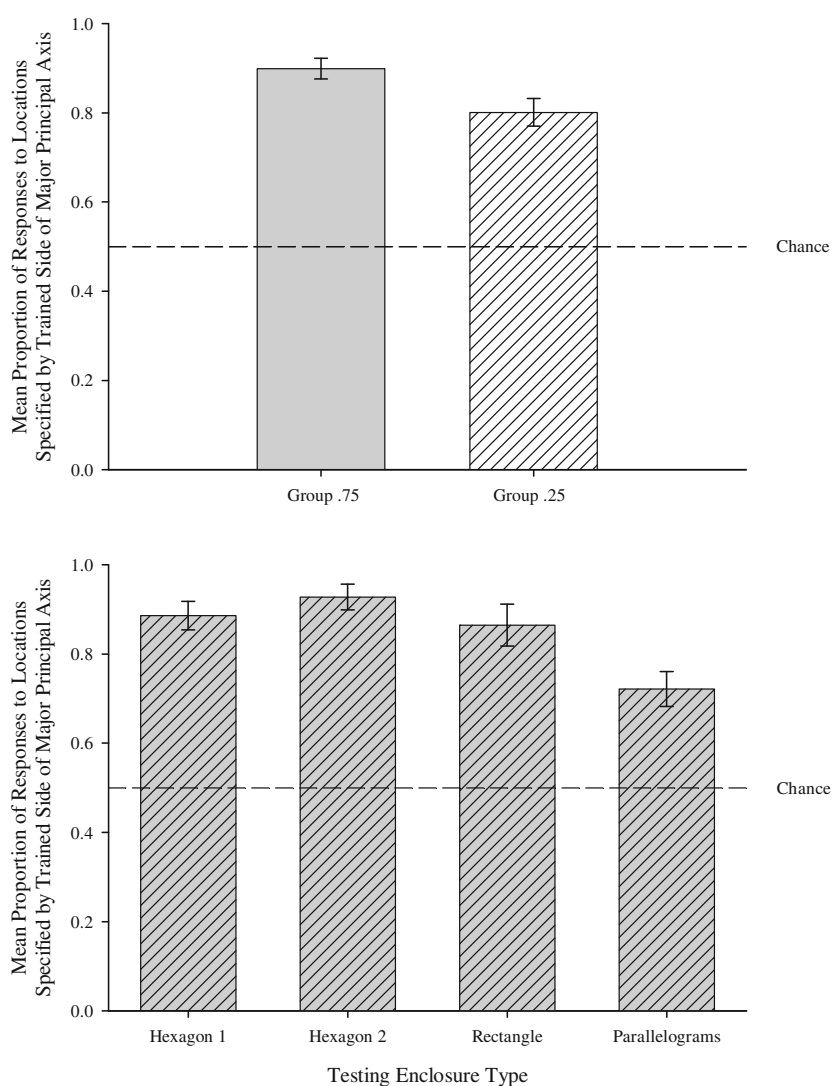

Fig. 3 Top panel: Mean proportion of responses to locations specified by the trained side of the major principal axis for both groups collapsed across enclosure types (main effect of group). Bottom panel: Mean proportion of responses to locations specified by the trained side of the major principal axis for each testing enclosure (main effect of enclosure type). Dashed lines represent chance performance. Error bars represent standard errors of the means

principal axis, as compared with Group .25. Figure 3 (bottom panel) shows the main effect of enclosure type; post hoc tests on the enclosure type factor revealed that the mean proportion of responses to locations specified by the trained side of the major principal axis was significantly lower for the parallelograms ( $p$ s $<.05$ ), as compared with all other enclosure types; none of the other enclosure types differed from each other ( $p \mathrm{~s}>.17)$. We suspect that the lower performance for the parallelograms, as compared with the other enclosure types, was due to the fact that in one of the parallelograms the locations specified by the trained side of the major principal axis were aligned with corner angles similar to those experience during training (i.e., acute or obtuse), whereas in the other parallelogram the locations specified by the trained side of the major principal axis were misaligned with corner angles similar to those experienced during training (i.e., acute or obtuse). Regardless, the mean proportion of responses to the locations specified by the trained side of the major principal axis was statistically greater than chance $(.50)$ for each enclosure, $t \mathrm{~s}(31)>5.64, p \mathrm{~s}<.001$, and suggests that participants primarily utilized the trained side of the major principal axis to determine orientation within each enclosure type.

\section{Discussion}

Results in the present dynamic three-dimensional virtual environment search task suggest that the discriminability of the major principal axis of an enclosure (and hence, its potential usefulness as an orienting cue) is a function of a relationship to the minor principal axis. Participants trained with a larger discriminability ratio (i.e., Group .75) responded to locations specified by the trained side of the major principal axis to a greater extent than did participants trained with a smaller discriminability ratio (i.e., Group .25), and such a result held across control trials and manipulations of shape. Importantly, our paradigm allowed us to control for the use of wall length or corner angle as the stimulus responsible for successful orientation (see Dawson et al., 2010; Miller \& Shettleworth, 2007; for a review, see Cheng, 2008) and to isolate the use of principal axis for orientation. Such isolation of the use of principal axis occurred because wall lengths and corner angles were rendered useless during testing in the novel enclosures.

Because our design allowed us to isolate the use of principal axes for orientation, we suggest that participants in both groups were primarily relying on the principal axes of space for orientation during control trials and across manipulations of enclosure shape. Such a conclusion is supported by the above-chance performance in all testing enclosures. Although it seems possible that participants in Group .25 may have been utilizing corner angles to a greater extent than did Group .75, such a strategy by participants in Group .25 should have influenced performance to a greater extent in enclosures containing corner angles similar to those experienced during training (i.e., hexagon 2 and parallelograms), as compared with enclosures in which those cues were absent (i.e., hexagon 1 and rectangle). In short, the use of corner angle for orientation should have produced a significant interaction. Given the lack of a significant interaction, we believe that the obtained results indicate that participants in both groups were utilizing the same spatial cue (i.e., principal axis) but simply utilized this spatial cue to a different extent.

One potential alternative explanation of the obtained difference between the groups during testing that need not appeal to a difference in the hypothetical discriminability ratio between major and minor principal axes relates to a difference between the groups with respect to enclosure size during training. Specifically, changes in enclosure size from training to testing have been shown to influence the relative contribution of feature and geometric cues in the 
reorientation process (Ratliff \& Newcombe, 2008; Sovrano, Bisazza, \& Vallortigara, 2005; 2007; Vallortigara, Feruglio, \& Sovrano, 2005). More specifically, geometric cues have been shown to exert a greater influence in smaller enclosures, whereas feature cues have been shown to exert a greater influence in larger enclosures (see also Miller, 2009). At first blush, it seems possible that the obtained difference between the groups during testing may have been due to a greater reliance on the principal axes during testing for participants trained within hexagon 1 , because of its relatively smaller size, as compared with hexagon 2.

Although we acknowledge such a possibility, existing evidence makes such an alternative explanation based on differences in size an unlikely account of the obtained differences between the groups in the present task. Primarily, evidence for the influence of enclosure size on the relative contribution of feature and geometric cues has come from tasks utilizing incidental learning paradigms. Specifically, these tasks involved training to both geometric cues and target objects such as beacons or landmarks, and these studies showed only a relative difference between use of feature and geometric cues during explicit conflict tests of the feature and geometric cues (Ratliff \& Newcombe, 2008; Sovrano et al., 2005, 2007; Vallortigara et al., 2005).

The present task was fundamentally different from these prior studies that have shown evidence for the influence of enclosure size on the relative contribution of feature and geometric cues. Primarily, the present task did not utilize an incidental learning paradigm because it did not involve training to target objects such as beacons or landmarks. Instead, participants in the present task were explicitly trained to geometric aspects of the enclosures, and the absence of target objects such as beacons or landmarks during training prohibited the possibility of any potential conflict between feature and geometric cues during testing. Importantly, there is evidence to suggest that geometric cues are utilized to an equivalent extent across manipulations of enclosure size in the absence of such conflict tests (see Sturz \& Kelly, 2009).

In short, there exist numerous differences between the task and those in prior studies that have obtained evidence for an influence of enclosure size. Whether the use of global (i.e., principal axis) versus local (i.e., wall lengths, angles) geometric cues is influenced by enclosure size in ways similar to those for feature (i.e., beacons, landmarks) versus geometric cues is open to further investigation, but these issues appear to fall beyond the ability of the present experiment to diagnose. Regardless, such issues do not appear to undermine an interpretation consistent with the discriminability of the major-to-the minor principal axis.

With respect to representations of space, our results provide further support that spatial coding is based on metric relations (Gallistel, 1990) and suggest that psychophysical principles may operate on the discrimination of parameters of environmental shape. Importantly, our results delineate a potential a priori measure of discriminability based on metric relations that may be utilized to predict the orientation ability of participants within an environment. For example, if one considers a square-shaped enclosure, the lengths of the major and minor principal axes are equivalent, and the resulting discriminability ratio would be 0 . Such a discriminability measure provides a potential explanation for the inability of participants to orient within such an enclosure (see Kelly \& Spetch, 2001; Sturz \& Kelly, 2009) and the diminished ability of participants trained or reared in such enclosures to orient within rectangular environments (Brown, Spetch, \& Hurd, 2007; Kelly, Spetch, \& Heth, 1998). Perhaps, much as for visual stimuli (Hubel \& Wiesel, 1963; LeVay, Wiesel, \& Hubel, 1980), early exposure to discriminable principal axes is critical for the development of orientation by these mechanisms. Minimally, our results suggest that surface-based orientation is influenced by a proportional relationship of principal axes and that psychophysical principles may operate on the discrimination of parameters of environmental shape. We suggest that these results delineate a testable mechanism for experiential and developmental changes in orientation ability and may assist in determining the mechanisms underlying surfacebased orientation.

Author's Note This research was conducted following the relevant ethical guidelines for human research. We thank Caroline Eastman, Kate Gaskin, and Scott Katz for assistance with data collection and scoring. We also thank three anonymous reviewers for their helpful comments on a previous version of the manuscript.

\section{References}

Brown, A. A., Spetch, M. L., \& Hurd, P. L. (2007). Growing in circles: Rearing environment alters spatial navigation in fish. Psychological Science, 18, 569-573.

Cheng, K. (1988). Some psychophysics of the pigeon's use of landmarks. Journal of Comparative Physiology A, 162, 815-826.

Cheng, K. (1989). The vector sum model of pigeon landmark use. Journal of Experimental Psychology. Animal Behavior Processes, $15,366-375$.

Cheng, K. (1990). More psychophysics of the pigeon's use of landmarks. Journal of Comparative Physiology A, 166, 857-864.

Cheng, K. (2005). Reflections on geometry and navigation. Connection Science, 17, 5-21.

Cheng, K. (2008). Whither geometry? Troubles of the geometric module. Trends in Cognitive Sciences, 12, 355-361.

Cheng, K., \& Gallistel, C. R. (2005). Shape parameters explain data from spatial transformations: Comment on Pearce et al. (2004) and Tommasi and Polli (2004). Journal of Experimental Psychology: Animal Behavior Processes, 31, 254-259.

Cheng, K., \& Newcombe, N. S. (2005). Is there a geometric module for spatial orientation? Squaring theory and evidence. Psychonomic Bulletin \& Review, 12, 1-23. 
Cheng, K., Srinivasan, M. V., \& Zhang, S. W. (1999). Error is proportional to distance measured by honeybees: Weber's law in the odometer. Animal Cognition, 2, 11-16.

Dawson, M. R. W., Kelly, D. M., Spetch, M. L., \& Dupuis, B. (2010). Using perceptrons to explore the reorientation task. Cognition, 14, 207-226.

Fechner, G. H. (1966). Elements of psychophysics (H. E. Adler, Trans). New York: Holt, Rinehart and Winston. (Original work published 1860)

Gallistel, C. R. (1990). The organization of learning. Cambridge: MIT Press.

Gescheider, G. A. (1997). Psychophysics: The fundamentals (3rd ed.). Mahwah: Erlbaum.

Hermer, L., \& Spelke, E. (1994). A geometric process for spatial representation in young children. Nature, 370, 57-59.

Hubel, D. H., \& Wiesel, N. (1963). Receptive fields of cells in striate cortex of very young, visually inexperienced kittens. Journal of Neurophysiology, 26, 994-1002.

Kelly, D. M., \& Spetch, M. L. (2001). Pigeons encode relative geometry. Journal of Experimental Psychology. Animal Behavior Processes, 27, 417-422.

Kelly, D. M., Spetch, M. L., \& Heth, C. D. (1998). Pigeons' (Columba livia) encoding of geometric and featuralproperties of a spatial environment. Journal of Comparative Psychology, 112, 259-269.

LeVay, S., Wiesel, T. N., \& Hubel, D. H. (1980). The development of ocular dominance columns in normal and visually deprived monkeys. The Journal of Comparative Neurology, 191, 1-51.

Miller, N. Y. (2009). Modeling the effects of enclosure size on geometry learning. Behavioural Processes, 80, 306-313.
Miller, N. Y., \& Shettleworth, S. J. (2007). Learning about environmental geometry: An associative model. Journal of Experimental Psychology. Animal Behavior Processes, 33, 191-212.

Ratliff, K. R., \& Newcombe, N. S. (2008). Reorienting when cues conflict: Evidence for an adaptive combination view. Psychological Science, 19, 1301-1307.

Rock, I. (1973). Orientation and form. New York: Academic.

Shelton, A. L., \& McNamara, T. P. (2001). Systems of spatial reference in human memory. Cognitive Psychology, 43, 274-310.

Sovrano, V. A., Bisazza, A., \& Vallortigara, G. (2005). Animals' use of landmarks and metric information to reorient: Effects of the size of the experimental space. Cognition, 97, 121-133.

Sovrano, V. A., Bisazza, A., \& Vallortigara, G. (2007). How fish do geometry in large and in small spaces. Animal Cognition, 10, 4754.

Sovrano, V. A., \& Vallortigara, G. (2006). Dissecting the geometric module: A sense linkage for metric and landmark information in animals' spatial reorientation. Psychological Science, 17, 616621.

Sturz, B. R., Gurley, T., \& Bodily, K. D. (2011). Orientation in trapezoid-shaped enclosures: Implications for theoretical accounts of geometry learning. Journal of Experimental Psychology. Animal Behavior Processes, 37, 246-253.

Sturz, B. R., \& Kelly, D. M. (2009). Encoding of relative enclosure size in a dynamic three-dimensional virtual environment by humans. Behavioural Processes, 82, 223-227.

Vallortigara, G., Feruglio, M., \& Sovrano, V. A. (2005). Reorientation by geometric and landmark information in environments of different sizes. Developmental Science, 8, 393-401. 